Algebraic $\&$ Geometric $\mathcal{T}_{\text {opology }}$

Volume 5 (2005) 1141-1172

Published: 18 September 2005

ATG

\title{
Nullification functors and the homotopy type of the classifying space for proper bundles
}

\author{
RAMÓN J. FLORES
}

\begin{abstract}
Let $G$ be a discrete group for which the classifying space for proper $G$-actions is finite-dimensional. We find a space $W$ such that for any such $G$, the classifying space $\underline{\mathrm{B}} G$ for proper $G$-bundles has the homotopy type of the $W$-nullification of $\mathrm{B} G$. We use this to deduce some results concerning $\underline{\mathrm{B}} G$ and in some cases where there is a good model for $\underline{\mathrm{B}} G$ we obtain information about the $\mathrm{B} \mathbb{Z} / p$-nullification of $\mathrm{B} G$.
\end{abstract}

AMS Classification 55P20; 55P 80

Keywords (Co)localization, finite groups, Eilenberg-MacLane spaces

\section{Introduction}

Let $G$ be a discrete group. We will say that a $G$-CW-complex $X$ is proper if the isotropy groups of the action are finite.

In 1971, J.P. Serre introduced in [37] the "classifying space for proper actions", that can be described as the unique proper $G$-CW-complex $\underline{\mathrm{E}} G$, up to $G$ homotopy, that enjoys the following universal property:

"If $X$ is another proper $G$-CW-complex, there exists a $G$-map $X \longrightarrow \underline{\mathrm{E} G}$ which is unique up to $G$-homotopy".

The space $\underline{\mathrm{E}} G$ appears as the principal new feature in the reformulation of the Baum-Connes conjecture stated in 3 by Baum-Connes-Higson. The conjecture, partially solved, asks if for a locally compact, Hausdorff and second countable group $G$, the assembly map from the Kasparov $K$-homology groups $K_{j}^{G}(\underline{\mathrm{E}} G)$ to the $C^{*}$-algebra $K$-theory groups $K_{j}\left(C_{r}^{*}(G)\right)$ is an isomorphism for $j=0,1$. The great amount of research that has emerged around this subject has led to a growing interest in the theory of proper actions.

An important part of the efforts carried out in this direction has been devoted to understand the relationship between the algebraic structure of $G$ and the 
homotopy-theoretic properties of $\underline{\mathrm{E}} G$ and its quotient space $\underline{\mathrm{E}} G / G$, which is currently denoted by $\underline{\mathrm{B}} G$. Probably the greatest success has been reached by interpreting correctly finiteness group-theoretic conditions over $G$ in order to build models of $\underline{\mathrm{E}} G$ enjoying various types of finiteness conditions. See ([28], section 5) for an excellent survey on this topic.

In the same way that happens with classical $G$-actions, the importance of $\underline{E} G$ and in particular of $\underline{\mathrm{B}} G$ does not come only because they reflect geometrically the algebraic properties of the group $G$, but because the importance of these spaces in the theory of $G$-bundles. Baum-Connes-Higson already pointed out that $\underline{\mathrm{B}} G$ classifes proper $G$-bundles (see [3], definition 8), and they described how to obtain them by making pullback on maps $X \longrightarrow \underline{\mathrm{B}} G$, a method of a clear classical flavor. Moreover, if $G$ is a group for which Baum-Connes conjecture holds, the knowledge of the homotopical structure of $\underline{\mathrm{B}} G$ plays a rôle in the computation of the $K$-theory of $C^{*}(G)$, and in general gives information about the integral homology of $G$. See ([28], section 8) for details about these results.

The most important attempt made so far to understand the homotopy type and properties of $\underline{\mathrm{B}} G$ is the paper of Leary-Nucinkis [24]. In it, the authors prove that for every CW-complex $X$ there exists a discrete group $G_{X}$ such that $\underline{\mathrm{B}} G_{X}$ is homeomorphic to $X$. This "Kan-Thurston-like" result is proved using essentially tools of the theory of graph of groups. As a by-product, they obtain a precise description of the fundamental group of $\underline{\mathrm{B}} G$ and a construction of $\underline{\mathrm{B}} G$ for some subgroups of right-angled Coxeter groups.

Although these results have been very useful for us (particularly the formula for the fundamental group), our approach to the homotopy type of $\underline{B} G$ has been different, and has been carried out with pure homotopy-theoretic tools. Our idea is to find a functor $F$ in the topological category that transforms models of $\mathrm{B} G$ on spaces that are homotopy equivalent to models of $\underline{\mathrm{B}} G$. This functor have enough good properties in order to read information about $\underline{\mathrm{B}} G$ from $\mathrm{B} G$ and vice versa.

The appropriate functor turns to be a nullification; a tool that was introduced by Bousfield in [4] in order to study periodic phenomena in unstable homotopy (in fact, he called it "periodization"), and that has been widely used since then. The utility of this functor in this context comes from the fact that it will allow us to apply all the machinery of localization developed in the 90's by Bousfield himself [4, 5], Dror-Farjoun [13], Chachólski 8] and others.

Our main result is the following:

Theorem 3.2 Let $G$ be a discrete group such that there exists a finitedimensional model for $\underline{\mathrm{B}} G$. Let $W_{\infty}$ denote $\bigvee \mathrm{B} \mathbb{Z} / p$, where the wedge is in- 
dexed by all primes. Then we have a homotopy equivalence $\mathbf{P}_{W_{\infty}} \mathrm{B} G \simeq \underline{\mathrm{B}} G$, where $\mathbf{P}_{W_{\infty}}$ denotes the $W_{\infty}$-nullification functor.

Observe that the condition concerning the existence of a model of $\underline{\mathrm{B}} G$ with finiteness conditions is not too restrictive, in account of the great quantity of groups that have recently appeared for which these conditions hold.

Now we describe in more detail the contents of each of the sections of the paper, and in particular we will comment a little bit the consequences of the main theorem.

In section 2 we review the needed background and results concerning proper actions, and we construct a particular model for the classifying space for families $E_{\mathcal{F}} G$ that will be very useful in the rest of the work; as a fundamental byproduct, we also obtain an appropriate model for $\mathrm{B}_{\mathcal{F}} G$.

Section 3 constitutes the bulk of the work, because it is devoted to the proof of the main theorem we stated before. The technique is the following: we apply the functor $\mathbf{P}_{W_{\infty}}$ to a suitable model of $\mathrm{B} G$, and we obtain that it is homotopy equivalent to the $W_{\infty}$-nullification of the nerve of some small category that only depends on $G$. This nerve turns out to be the model built in the previous section for $\underline{\mathrm{B}} G$, and we finish by checking that in the conditions of the theorem $\underline{\mathrm{B}} G$ is $W_{\infty}$-null.

The rest of the paper is devoted to take out some consequences of the main theorem. So, in section 4 we describe the behaviour of the functor $\underline{B}$ with respect to various fundamental constructions in homotopy theory, namely products, wedges or colimits. Moreover, we identify in some cases the universal cover of $\underline{\mathrm{B}} G$ and we obtain some conditions about preservation of fibrations under passing to classifying spaces.

We begin the following section with a short new proof of the well-known fact that if $G$ is a locally finite group which cardinal is smaller than $\aleph_{\omega}$, $\underline{\mathrm{B}} G$ is contractible (indeed, the statement is true for all locally finite groups, see [11]). Later, we treat the case of groups for which the normalizer condition holds, an ample class of discrete groups that include, for example, all the nilpotent groups. We prove that if a group $G$ in this class admits a finite-dimensional model for $\underline{\mathrm{B}} G$, then $\underline{\mathrm{B}} G \simeq \mathrm{B} H$ for some quotient group $H$ that we identify. In particular, in this case $\underline{\mathrm{B}} G$ is nilpotent as a space if $G$ is nilpotent as a group. We finish this paragraph by studying the $\mathrm{B} \mathbb{Z} / p$-nullification of classifying spaces of supersoluble groups.

In section 6 we take the opposite point of view, showing that the main theorem can give information in the two directions. More concretely, we focus our attention on groups of isometries of the real plane, and taking profit of well-known 
geometric properties of them we obtain via $\underline{\mathrm{B}} G$ a lot of information on the $\mathrm{B} \mathbb{Z} / p$-nullification of their classifying spaces.

Last section is devoted to the study of a canonical map $\mathrm{B} G \longrightarrow \mathrm{B}_{\mathcal{F}} G$ (defined previously) that always relates the classical and proper classifying spaces. More concretely, we prove that the homotopy fiber of that map can be described as a homotopy colimit of classifying spaces of groups of $\mathcal{F}$ over a contractible category. We finish by proving a technical and interesting statement that appears in the proof and concerns the localization of a comma category.

Acknowledgements I wish to thank Carles Broto, for turning my attention to proper actions and the world of Geometric Group Theory, and for all the time we have spent discussing about these topics. The results about crystallographic groups were motivated by a suggestion of Ian Leary, whom I acknowledge his interest in my work. I am also grateful to Emmanuel Dror-Farjoun, who pointed out some very useful observations that enriched the results of last section. Finally, I would like to thank the Institute Galilée, Université Paris XIII, for their hospitality in the seven months I spent there, and the referee, for the detailed report and numerous suggestions. The author is partially supported by MCYT grant BFM2001-2035.

\section{Useful models for $\mathbf{E}_{\mathcal{F}} G$ and $\mathbf{B}_{\mathcal{F}} G$}

We will begin by recalling the definition of the classifying space for families. Further information about proper actions and their classifying spaces can be found in [3, 24, 33, or [12].

Definition 1 Suppose $\mathcal{F}$ is a family of subgroups of a discrete group $G$ that is closed under conjugation and taking subgroups. We will say that a $G$-CWcomplex $Y$ is a model for $E_{\mathcal{F}} G$ if the isotropy group of each point belongs to $\mathcal{F}$ and for each $H \in \mathcal{F}$, the fixed-point space $Y^{H}$ is contractible.

The $G$-space $\mathrm{E}_{\mathcal{F}} G$ is characterized by the following universal property:

Proposition 2.1 If $X$ is a model for $\mathrm{E}_{\mathcal{F}} G$, then for each $G$-CW-complex $Y$ whose isotropy groups lie in $\mathcal{F}$ there is a map $Y \longrightarrow X$ which is unique up to $G$ homotopy. Moreover, two models for $\mathrm{E}_{\mathcal{F}} G$ are always $G$-homotopy equivalent. Conversely, if $X$ is a $G$-CW-complex for which this universal property holds, $X$ is a model for $\mathrm{E}_{\mathcal{F}} G$. 
The quotient space $\mathrm{E}_{\mathcal{F}} G / G$ is usually denoted by $\mathrm{B}_{\mathcal{F}} G$. As $\mathrm{E}_{\mathcal{F}} G$ is unique up to $G$-homotopy equivalence, $\mathrm{B}_{\mathcal{F}} G$ is unique up to homotopy equivalence; see (24, section 2) for a review of the main properties of $\mathrm{B}_{\mathcal{F}} G$.

If there is no explicit mention against it, we will suppose from here that $\mathcal{F}$ is the family of finite subgroups of $G$. In this case, it is standard to denote $\mathrm{E}_{\mathcal{F}} G$ and $\mathrm{B}_{\mathcal{F}} G$ by $\underline{\mathrm{E}} G$ and $\underline{\mathrm{B}} G$, respectively. In particular, it can be seen that every group homomorphism $G \longrightarrow G^{\prime}$ induces a $G$-map $\underline{\mathrm{E}} G \longrightarrow \underline{\mathrm{E}} G^{\prime}$ (respectively a map $\underline{\mathrm{B}} G \longrightarrow \underline{\mathrm{B}} G^{\prime}$ ) which is unique up to $G$-homotopy (respectively up to homotopy).

A number of geometric constructions for $\mathrm{E}_{\mathcal{F}} G$ and its quotient space is available in the literature (see section 4 of [27] for a description of some of them), but to describe the relationship between $\mathrm{B} G$ and $\mathrm{B}_{\mathcal{F}} G$ we will need to build these spaces as nerves of small categories. This is the main goal of this section.

Recall first the definition of the orbit category associated to a group $G$ and a family of subgroups $\mathcal{F}$ : the objects are the homogeneous spaces $G / H$, with $H \in \mathcal{F}$, and the morphisms are the $G$-maps. It is not hard to see that there is a bijective map

$$
\operatorname{Mor}(G / K, G / H)=\left\{g \in G \mid g^{-1} K g \subseteq H\right\} / H
$$

given by $f \longrightarrow f(e K)$, where $e$ is the identity element of $G$. The key definition we need for building the desired model of $\mathrm{E}_{\mathcal{F}} G$ is the following (see [15], section 2, for details):

Definition 2 Let $\mathcal{D}$ be a small category, Cat the category where the objects are the small categories and whose morphisms are functors, and $f: \mathcal{D} \rightarrow$ Cat a functor. The Grothendieck construction $\mathbf{G r}(f)$ associated to $f$ is defined as the category whose objects are the pairs $(d, x)$, with $x \in \mathcal{D}$ and $x \in f(d)$, and where a morphism $(d, x) \rightarrow\left(d^{\prime}, x^{\prime}\right)$ is a pair $(u, v)$ where $u: d \rightarrow d^{\prime}$ is a morphism in $\mathcal{D}$ and $v: f(u)(x) \rightarrow x^{\prime}$ is a morphism in $f\left(d^{\prime}\right)$. The composition is made in the obvious way.

The main feature of this construction, due to Thomason, is the following:

Theorem 2.2 Let $\mathcal{D}$ be a small category, $F: \mathcal{D} \longrightarrow$ Cat a functor, and $\operatorname{Gr}(F)$ the Grothendieck construction of $F$. Then there exists a natural weak homotopy equivalence:

$$
\mathrm{N}(\mathbf{G r}(F)) \simeq \text { hocolim } \mathrm{N}(F)
$$

where $\mathrm{N}$ denotes the nerve. 
Proof See 39, 1.2.

Now we can describe our model of the universal space $\mathrm{E}_{\mathcal{F}} G$.

Proposition 2.3 Let $G$ be a discrete group. Consider the functor

$$
R: \mathbf{O}_{\mathcal{F}} \longrightarrow \text { Cat }
$$

that sends every homogeneous space $G / H$ to the category $G / H$ (whose objects are the elements of $G / H$ and there is only identity morphisms), and the morphisms to the obvious functors. In these conditions, we have that the nerve of the Grothendieck construction of $f$ is a model for $\mathrm{E}_{\mathcal{F}} G$.

Proof For convenience, we will denote $X=|\mathrm{N}(\mathbf{G r}(R))|$. This space has a natural action of $G$ given by the left action of $G$ in every homogeneous space $G / H$. We will prove firstly that for every $x \in X$ the isotropy group $G_{x}$ of $x$ belongs to $\mathcal{F}$.

The action of $G$ over $X$ is simplicial and is induced from the action of $G$ over the homogeneous spaces, so by definition of nerve we will only need to study the action of $G$ over the vertices of $X$. Hence, let $(G / H, a)$ be the pair associated with the vertex $x$. It is clear that

$$
G_{x}=\{g \in G \mid g a H=a H\}=\{g \in G \mid \exists h \in H \text { s. t. } g a h=a\}
$$

and this amounts to say that $g \in a H a^{-1}$. So, $G_{x}=a H a^{-1}$, that belongs to $\mathcal{F}$ because $H$ does.

Now we will see that for every $K \in \mathcal{F}$, the set of fixed points $X^{K}$ is contractible. Now, a point on a simplex is fixed by a subgroup if and only if all vertices are fixed, because the action of $G$ is defined over the vertices and then extended to the rest of the simplices by linearity.

So, consider a vertex $x \in X$. This point is fixed by the action of $K$ if, given the pair $(G / H, a H)$ associated to it, we have that, for every $k \in K, k a H=a H$. Thus, we see that

$$
X^{K}=\bigcup_{H \in \mathcal{F}}\left\{(G / H, a H) \mid a^{-1} K a \subseteq H\right\} / H .
$$

So, for every element $(G / H, a H) \in X^{K}$ there exists one and only one morphism $(G / K, e K) \longrightarrow(G / H, a H)$, or in other words, $X^{K}$ can be identified with the nerve of the undercategory (in the sense of [30, II.6) associated to the element $(G / K, e K)$ of the category $\mathbf{G r}(R)$. This nerve is contractible, and then $X^{K}$ is contractible too. So we are done. 
Note that if we consider the action of $G$ over $\operatorname{Gr}(R)$ via functors, the objects of $\operatorname{Gr}(R) / G$ are the homogeneous spaces $G / H, H<G$ finite, and the morphisms are the $G$-maps. So, this quotient category is identified in a natural way with the orbit category $\mathbf{O}_{\mathcal{F}}$, and in particular $\mathrm{N}\left(\mathbf{O}_{\mathcal{F}}\right)$ is a model for $\mathrm{B}_{\mathcal{F}} G$.

Remark 2.4 Observe that if we take the realizations of the nerves of $\boldsymbol{G r}(R)$ and $\mathbf{O}_{\mathcal{F}}$, we obtain models of $E_{\mathcal{F}} G$ and $B_{\mathcal{F}} G$ in the category of (topological) spaces.

Remark 2.5 The idea of the construction comes from ([2], section 2), although they only describe it in the case of $G$ finite, being $\mathcal{F}$ the family of finite subgroups of $G$, and with another purpose. In the language of that paper, we have proved that $X$ is the $\mathcal{F}$-approximation to a point.

We conclude this section with a modification of the previous models that will be useful in the sequel. So, if $\left\{H_{1}, \ldots, H_{n}\right\}$ are subgroups of $G$ that belong to $\mathcal{F}$ and such that for every $0<i<n$ there exists a $G$-equivariant map $G / H_{i} \longrightarrow G / H_{i+1}$, we define $\mathbf{G} / \mathbf{H}_{\mathbf{1}} \longrightarrow \ldots \longrightarrow \mathbf{G} / \mathbf{H}_{\mathbf{n}}$ as the small category whose elements are $n$-uples $\left(a_{1} H_{1}, \ldots, a_{n} H_{n}\right)$ such that for every $0<i<n$ there exists a $G$-equivariant map $f_{i}: G / H_{i} \longrightarrow G / H_{i+1}$ with $f_{i}\left(a_{i} H_{i}\right)=$ $a_{i+1} H_{i+1}$, and whose morphisms are the identity maps. Now, if $\boldsymbol{\Gamma}$ is the poset category of non-degenerate simplices of $\mathrm{N}\left(\mathbf{O}_{\mathcal{F}}\right)$, we define a functor $S: \boldsymbol{\Gamma}^{o p} \longrightarrow$ Cat that takes the simplex represented by the chain of maps $\left\{G / H_{1} \longrightarrow \ldots \longrightarrow\right.$ $\left.G / H_{n}\right\}$ to the category $\mathbf{G} / \mathbf{H}_{\mathbf{1}} \longrightarrow \ldots \longrightarrow \mathbf{G} / \mathbf{H}_{\mathbf{n}}$, and the face maps to the obvious functors. Then we have the following:

Proposition 2.6 In the previous conditions, $\operatorname{hocolim}_{\Gamma} \mathrm{N}(S)$ is a model for $\mathrm{E}_{\mathcal{F}} G$, and $\mathrm{N}(\boldsymbol{\Gamma})$ is a model for $\mathrm{B}_{\mathcal{F}} G$.

Proof By theorem 2.2. hocolim $\boldsymbol{\Gamma}^{\mathrm{N}}(S) \simeq \mathrm{N}(\mathbf{G r}(S))$, and on the other hand, the left action of $G$ over every homogeneous space $G / H,(H \in \mathcal{F})$ induces, via functors, another one over the categories $\mathbf{G} / \mathbf{H}_{\mathbf{1}} \longrightarrow \ldots \longrightarrow \mathbf{G} / \mathbf{H}_{\mathbf{n}}$. Now, observe that $\mathrm{N}(\mathbf{G r}(S))$ is the subdivision (in the sense of [19], III.4) of $\mathrm{N}(\mathbf{G r}(R)$ ), where $R$ is the functor defined in proposition 2.3. In fact, if we take a nondegenerate simplex of $\mathrm{N}(\mathbf{G r}(R))$ that is represented by a chain of morphisms $\left(G / H_{1}, a_{1} H_{1}\right) \longrightarrow \ldots \longrightarrow\left(G / H_{n}, a_{n} H_{n}\right)$, its barycenter is the vertex of the nerve $\mathrm{N}(\mathbf{G r}(S))$ represented by the object $\left(\mathbf{G} / \mathbf{H}_{\mathbf{1}} \longrightarrow \ldots \longrightarrow \mathbf{G} / \mathbf{H}_{\mathbf{n}}, a_{1} H_{1}\right.$ $\left.\longrightarrow \ldots \longrightarrow a_{n} H_{n}\right)$ of $\mathbf{G r}(S)$. Then, by (21] prop. 12-14), there exists a homotopy equivalence $|\mathrm{N}(\mathbf{G r} R)| \simeq|\mathrm{N}(\mathbf{G r} S)|$, that is a $G$-equivalence by construction, and then $\mathrm{N}(\mathbf{G r} S)$ is a model for $\mathrm{E}_{\mathcal{F}} G$. A similar line of reason proves that $\mathrm{N}(S)$ is a model for $\mathrm{B}_{\mathcal{F}} G$. 
The main advantage of these models is that they reconstruct $\mathrm{E}_{\mathcal{F}} G$ and $\mathrm{B}_{\mathcal{F}} G$ as homotopy colimits over a poset category, and in particular they have structure of simplicial complexes. These facts will be very useful in the final section.

\section{$3 \underline{\mathrm{B}} G$ is homotopy equivalent to a nullification}

Let $G$ be a discrete group, $\mathcal{F}$ a family of subgroups of $G$ closed under conjugation and taking subgroups. As a first step in our study of the relation between the classical and proper classifying spaces, we will describe a canonical map that always relates $\mathrm{B} G$ and $\mathrm{B}_{\mathcal{F}} G$.

So, consider models of $\mathrm{E} G$ and $\mathrm{E}_{\mathcal{F}} G$; both of them are $G$-spaces, and then we can make the Borel construction $\mathrm{E} G \times_{G} \mathrm{E}_{\mathcal{F}} G$. Now, let $p_{1}$ be the projection

$$
\mathrm{E} G \times{ }_{G} \mathrm{E}_{\mathcal{F}} G \stackrel{p_{1}}{\longrightarrow} \mathrm{E} G / G \simeq \mathrm{B} G .
$$

The action of $G$ over $\mathrm{E} G$ is free, so the map $p_{1}$ is a fibration, and its homotopy fiber $\mathrm{E}_{\mathcal{F}} G$ is contractible. Thus, $p_{1}$ is a homotopy equivalence, and $\mathrm{E} G \times{ }_{G} \mathrm{E}_{\mathcal{F}} G$ is a model of $\mathrm{B} G$.

Consider now the projection over the second component

$$
\mathrm{E} G \times_{G} \mathrm{E}_{\mathcal{F}} G \stackrel{p_{2}}{\longrightarrow} \mathrm{E}_{\mathcal{F}} G / G \simeq \mathrm{B}_{\mathcal{F}} G .
$$

We have seen that $\mathrm{E} G \times_{G} \mathrm{E}_{\mathcal{F}} G$ and $\mathrm{E}_{\mathcal{F}} G / G$ are respectively models for $\mathrm{B} G$ and $\mathrm{B}_{\mathcal{F}} G$, and then $p_{2}$ can be thought as a map $\mathrm{B} G \longrightarrow \mathrm{B}_{\mathcal{F}} G$ that we will call $f$ in the rest of the section. The map $f$ is not a fibration in general, because the action of $G$ over $\mathrm{E}_{\mathcal{F}} G$ is not free. In fact, if $x \in \mathrm{B}_{\mathcal{F}} G$, we have that $f^{-1}(x)$ has the homotopy type of $\mathrm{E} G \times{ }_{G} G / H_{x}$, being $H_{x}$ the isotropy group of $x$, that belongs to $\mathcal{F}$. Now, $\mathrm{E} G \times{ }_{G} G / H_{x}$ is a model for $\mathrm{B} H_{x}$, and hence all the fibers of the map $f$ have the homotopy type of classifying spaces of groups of $\mathcal{F}$.

This fact gave us the intuition that the map we have studied could encode a functorial way of passing from the usual classifying space of $G$ to the classifying space for proper $G$-bundles, and what is more important, to obtain valuable information of the latter starting from the homotopy structure of $\mathrm{B} G$, and viceversa. More concretely, we searched for a functor $F$ such that the following conditions hold:

(1) $F$ "kills" the homotopy fiber of $f$.

(2) $F(f)$ is a weak equivalence. 


$$
F(\underline{\mathrm{B}} G) \simeq \underline{\mathrm{B}} G .
$$

The two first conditions give the impression, according to (13], 1.H.1 and 3.D.3), of $F$ being a localization functor $L$ in the sense of Dror-Farjoun, and in fact, the functor we have found has been the $A$-nullification functor with respect to a certain space $A$. For the main properties of these functors you can look at 4, [8] or [13, although we will recall here the definition.

Let $A$ and $X$ be spaces; $X$ is said $A$-null if the mapping space $\operatorname{map}(A, X)$ is homotopy equivalent to $X$ via the inclusion of constant maps $X \longrightarrow \operatorname{map}(A, X)$. The $A$-nullification of $X$ is a functor $\mathbf{P}_{A}$ : Spaces $\longrightarrow$ Spaces that takes every space $X$ to an $A$-null space $\mathbf{P}_{A} X$ such that there exists a universal map $X \longrightarrow \mathbf{P}_{A} X$ which induces a weak homotopy equivalence

$$
\operatorname{map}\left(\mathbf{P}_{A} X, Y\right) \simeq \operatorname{map}(X, Y)
$$

for every $A$-null space $Y$. It can be seen that any other $A$-null space $X$ for which the last property holds is indeed homotopy equivalent to $\mathbf{P}_{A} X$. So, we have defined a functor in the category of unpointed spaces (that can also be defined in the category of pointed spaces, although we we will only work here with unpointed spaces), which is always coaugmented and idempotent, and kills the structure of $X$ that "depends" on $A$. In fact, $\mathbf{P}_{A} X$ is the localization of $X$ with respect to the constant map $A \longrightarrow *$, and the spaces $X$ for which $\mathbf{P}_{A} X$ is contractible are called $A$-acyclic.

Consider now the set of all prime numbers $\left\{p_{1}, p_{2}, p_{3} \ldots\right\}$ with the usual order, and let $X$ be a space. In the remaining of the paper we will denote by $W_{n}$ the space $\mathrm{B} \mathbb{Z} / p_{1} \vee \ldots \vee \mathrm{B} \mathbb{Z} / p_{n}$, and by $W_{\infty}$ the wedge $\bigvee \mathrm{B} \mathbb{Z} / p$ extended over of the all prime numbers. The next key lemma is one strong reason that suggests that the $W_{\infty}$-nullification is the functor we need.

Theorem 3.1 If $G$ is a finite group, then $\mathbf{P}_{W_{\infty}} \mathrm{B} G$ is contractible.

Proof A point is always null, so we only need to prove that for every $W_{\infty}$-null space $X$ there is an equivalence $X \simeq \operatorname{map}\left(\mathbf{P}_{W_{\infty}} \mathrm{B} G, X\right)$. But the $W_{\infty}$-null spaces are, in particular, $W_{n}$-null for every $n$; hence,

$$
\operatorname{map}\left(\mathbf{P}_{W_{\infty}} \mathrm{B} G, X\right) \simeq \operatorname{map}(\mathrm{B} G, X) \simeq \operatorname{map}\left(\mathbf{P}_{W_{n}} \mathrm{~B} G, X\right)
$$

for every $n$. Now, suppose that $|G|=p_{j_{1}}^{n_{1}} p_{j_{2}}^{n_{2}} \ldots p_{j_{m}}^{n_{m}}$, with $j_{1}<\ldots j_{m}$. Because of $\left([17,3.3)\right.$, we know that $\mathbf{P}_{W_{k}} \mathrm{~B} G$ is contractible for every $k \geq j_{m}$. This implies

$$
\operatorname{map}\left(\mathbf{P}_{W_{\infty}} \mathrm{B} G, X\right) \simeq \operatorname{map}\left(\mathbf{P}_{W_{n}} \mathrm{~B} G, X\right) \simeq X
$$

as we claimed. 
Now suppose that $G$ is a discrete group; we are in position of stating our main theorem:

Theorem 3.2 Let $G$ be a group such that there exists a finite-dimensional model for $\underline{\mathrm{B}} G$, and let $W_{\infty}$ be as before; we have that $\underline{\mathrm{B}} G$ is homotopy equivalent to $\mathbf{P}_{W_{\infty}} \mathrm{B} G$.

Proof Consider the model of $\underline{E} G$ given in section 2 as the realization of the nerve of the Grothendieck construction of a functor

$$
R: \mathbf{O}_{\mathcal{F}} \longrightarrow \mathrm{Cat}
$$

described there. We have seen that $\mathrm{E} G \times{ }_{G} \underline{\mathrm{E}} G$ is a model of $\mathrm{B} G$, so using theorem 2.2 we obtain

$$
\mathrm{B} G \simeq \mathrm{E} G \times_{G} \underline{\mathrm{E}} G \simeq \mathrm{E} G \times_{G}\left(\text { hocolim }_{\mathbf{O}_{\mathcal{F}}} R\right) \simeq \operatorname{hocolim}_{\mathbf{O}_{\mathcal{F}}}\left(\mathrm{E} G \times_{G} R(-)\right)
$$

where the previous equivalence is a simple application of $([16,6.5)$. Now, if we apply the nullification functor $\mathbf{P}_{W_{\infty}}$ to the previous string of equivalences, we obtain a weak equivalence $\mathbf{P}_{W_{\infty}} \mathrm{B} G \simeq \mathbf{P}_{W_{\infty}}$ hocolim $_{\mathbf{O}_{\mathcal{F}}}\left(\mathrm{E} G \times_{G} R(-)\right)$, and the latter is equivalent, by (13]1.H.1), to $\mathbf{P}_{W_{\infty}}$ hocolim $\mathbf{O}_{\mathcal{F}} \mathbf{P}_{W_{\infty}}\left(\mathrm{E} G \times_{G} R(-)\right)$. Observe that the spaces that appear in the target of the functor

$$
\mathrm{E} G \times{ }_{G} \mathrm{~N}(R(-)): \mathbf{O}_{\mathcal{F}} \longrightarrow \text { Spaces }
$$

have the homotopy type of classifying spaces of finite subgroups of $G$. Hence, if we apply the previous proposition, we have that $\mathbf{P}_{W_{\infty}} \circ\left(\mathrm{E} G \times_{G} R(-)\right)$ is equivalent to the constant functor, and then

$$
\mathbf{P}_{W_{\infty}} \mathrm{B} G \simeq \mathbf{P}_{W_{\infty}} \operatorname{hocolim}_{\mathbf{O}_{\mathcal{F}} *} \simeq \mathbf{P}_{W_{\infty}}\left(\mathrm{N}\left(\mathbf{O}_{\mathcal{F}}\right)\right) \simeq \mathbf{P}_{W_{\infty}}(\underline{\mathrm{B}} G) .
$$

Then, by the solution of Miller to the Sullivan conjecture [31, we know that the space $\operatorname{map}\left(W_{\infty}, \underline{\mathrm{B}} G\right)$ is homotopy equivalent to $\underline{\mathrm{B}} G$, and hence $\underline{\mathrm{B}} G$ is $W_{\infty}$-null. This means that $\mathbf{P}_{W_{\infty}} \underline{\mathrm{B}} G$ is homotopy equivalent to $\underline{\mathrm{B}} G$, and we are done.

The following generalization, that will be widely applied in section 6 , is an immediate consequence of the proof of the previous theorem:

Corollary 3.3 If $\mathcal{F}$ is a family of finite subgroups of $G$ closed under conjugation and taking subgroups, and $\mathbf{P}_{A}$ is a nullification functor such that $\mathbf{P}_{A} \mathrm{~B} H \simeq *$ for every $H \in \mathcal{F}$, then the map $f: \mathrm{B} G \longrightarrow \mathrm{B}_{\mathcal{F}} G$ is an equivalence after $A$-nullification.

If we particularize for the family of all the finite groups, we obtain the following: 
Corollary 3.4 If $G$ is a discrete group, the classifying spaces $\mathrm{B} G$ and $\underline{\mathrm{B}} G$ are always equivalent after $W_{\infty}$-nullification; moreover, the map $f$ that was described at the beginning of this section is, in fact, equivalent to the $W_{\infty}$ nullification map if $G$ admits a finite-dimensional model for $\underline{\mathrm{B}} G$.

Proof The fact that $\mathrm{B} G$ and $\mathrm{B}_{\mathcal{F}} G$ are $\mathbf{P}_{W_{\infty}}$-equivalent is a particular case of the previous corollary. For the second statement, if we have the fibration

$$
\mathbf{F i b} f \longrightarrow \mathrm{B} G \longrightarrow \mathrm{B}_{\mathcal{F}} G
$$

(where Fibf stand for the homotopy fibre of $f$ ), the base is $W_{\infty}$-null, and then by $([13], 3 . D .3)$ the fibration is preserved under $W_{\infty}$-nullification. Now the result is an easy consequence of $([8,14.2)$.

It is worth to point out that the finiteness conditions under which the main theorem holds is that there exists a model of $\underline{\mathrm{B}} G$ for which $\operatorname{map}_{*}\left(W_{\infty}, \underline{\mathrm{B}} G\right)$ is weakly contractible. This is weaker than having a finite-dimensional model for $\underline{\mathrm{B}} G$, but the condition that will always hold for the groups that appear in the rest of this note will be the latter, because it is the usual one that appears in the literature of Geometric Group Theory. It would be interesting to find cohomological conditions to be a Miller space (that is, spaces $X$ for which $\operatorname{map}(\mathrm{BZ} / p, X) \simeq X)$ for all the primes at the same time, because we could then apply the theorem to these spaces. Recall that there already exist wellknown cohomological conditions of this kind for isolated primes, as for example the Lannes-Schwartz Theorem [23].

On the other hand, we cannot expect that theorem 3.2 holds for any $G$. To see this, take a space $X$ which is not $W_{\infty}$-null. According to [24], there exists a discrete group $G$ such that $X$ is a model for $\underline{B} G$; so our result does not hold for $G$.

We finish this section by showing that the discreteness of $G$ is necessary in theorem 3.2 .

Example 3.5 Let us consider the classifying space of $S^{1}$. As the circle is compact, the classifying space for proper $S^{1}$-bundles is a point by definition. On the other hand, consider the rationalization map $B S^{1} \longrightarrow K(\mathbb{Q}, 2)$. By the homotopy long exact sequence, the homotopy fibre of this map has the homotopy type of $K\left(\oplus \mathbb{Z}_{p^{\infty}}, 1\right)$, where the direct sum runs over all primes. As every Prüfer group $\mathbb{Z}_{p^{\infty}}$ is a colimit of a telescope of injections between $p$ groups, the results ([13] 1.D.3) and ([17, 3.3) imply that $\mathbf{P}_{W_{\infty}} K\left(\oplus \mathbb{Z}_{p^{\infty}}, 1\right)$ is 
contractible. Then, by (13 1.H.1) the rationalization is preserved by $W_{\infty}$ nullification, and as $K(\mathbb{Q}, 2)$ is clearly $W_{\infty}$-null, we have that $\mathbf{P}_{W_{\infty}} B S^{1} \simeq$ $K(\mathbb{Q}, 2)$, which is non-contractible. In fact, it seems plausible to conjecture that the $W_{\infty}$-nullification of the classifying space of a compact Lie group is homotopy equivalent to its rationalization.

\section{The homotopy type of $\underline{\mathrm{B}} G$}

In this paragraph we are going to prove some interesting consequences that the main theorem 3.2 has over the homotopy type of $\underline{B} G$. Essentially, the idea is to use properties of the nullification functors for describing the classifying space for $G$-proper bundles.

Remark 4.1 From now on, we particularize for the case $\mathcal{F}$ being the family of all the finite subgroups of $G$, although a great part of the results we obtain in the next sections remains valid for any subfamily of $\mathcal{F}$ that is subgroup-closed and conjugation-closed. Even if we do not mention it, we will also suppose that the finiteness conditions of theorem 3.2 hold for all the groups that appear in this section.

We begin by analyzing the behaviour of the functor $\underline{B}$ under products.

Proposition 4.2 Let $G_{1}$ and $G_{2}$ discrete groups which possess a finite dimensional model for the classifying space for proper bundles. Then the following holds:

- A model for $\underline{\mathrm{B}}\left(G_{1} \times G_{2}\right)$ is given by $\underline{\mathrm{B}} G_{1} \times \underline{\mathrm{B}} G_{2}$.

- The wedge $\underline{\mathrm{B}} G_{1} \vee \underline{\mathrm{B}} G_{2}$ is a model for $\underline{\mathrm{B}}\left(G_{1} * G_{2}\right)$.

Proof It is known that $\mathrm{B}\left(G_{1} \times G_{2}\right) \simeq \mathrm{B} G_{1} \times \mathrm{B} G_{2}$. Using that $\underline{\mathrm{B}} G_{1} \times \underline{\mathrm{B}} G_{2}$ is $W_{\infty}$-null (because the finiteness) and the preservation property ([13] 1.A.8, prop. 4), we obtain that

$\underline{\mathrm{B}}\left(G_{1} \times G_{2}\right) \simeq \mathbf{P}_{W_{\infty}}\left(\underline{\mathrm{B}}\left(G_{1} \times G_{2}\right)\right) \simeq \mathbf{P}_{W_{\infty}}\left(\underline{\mathrm{B}} G_{1}\right) \times \mathbf{P}_{W_{\infty}}\left(\underline{\mathrm{B}} G_{2}\right) \simeq \underline{\mathrm{B}} G_{1} \times \underline{\mathrm{B}} G_{2}$.

The proof of the second statement is similar, using that $\mathrm{B}\left(G_{1} * G_{2}\right) \simeq \mathrm{B} G_{1} \vee \mathrm{B} G_{2}$ and recalling that we can apply $([13,1 . D .5)$ because a wedge of a special case of pointed homotopy colimit. 
It is worth to point out that these results are established in $(28,4.9)$ without any assumption on the dimension, although this way of proving them is probably new. On the other hand, the second of them can be generalized to other colimits, like some telescopes and pushouts.

Proposition 4.3 Let $\left\{G_{i}\right\}_{i \in \mathbb{N}}$ be a collection of discrete groups which possess a finite-dimensional model for the classifying space for proper bundles . Then the following holds:

- If we have the pushout of groups

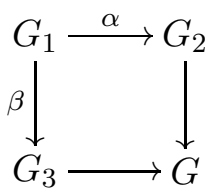

and the homomorphisms $\alpha$ and $\beta$ are injective, then the pushout of the induced diagram of classifying spaces for proper $G$-bundles is a model for $\underline{B} G$.

- If $G_{1} \longrightarrow G_{2} \longrightarrow G_{3} \longrightarrow \ldots$ is a telescope of groups where the maps are injective and we denote by $G$ the colimit of the telescope, we have that the colimit of the telescope induced by $\underline{\mathrm{B}}$ is a model for $\underline{\mathrm{B}} G$.

Proof To prove the first statement, recall that by Whitehead's theorem ([7], II.7.3) the pushout of the classical classifying spaces is the classifying space of the pushout. As the inclusions $\mathrm{B} G_{1} \hookrightarrow \mathrm{B} G_{2}$ and $\mathrm{B} G_{1} \hookrightarrow \mathrm{B} G_{3}$ are cofibrations, $\mathrm{B} G$ has the homotopy type of the homotopy pushout. If we apply now the functor $\mathbf{P}_{W_{\infty}}$ to the diagram, the result is deduced from theorem [3.2. ([13], 1.D.3) and the fact that there exists a finite-dimensional model for the homotopy pushout of the induced diagram

$$
\underline{\mathrm{B}} G_{2} \longleftarrow \underline{\mathrm{B}} G_{1} \longrightarrow \underline{\mathrm{B}} G_{3} .
$$

The second statement can be proved in an analogous way using again the relationship between localization and colimits given in ([13], 1.D.3) and the fact that the strict colimit of a telescope of cofibrations has always the homotopy type of the homotopy colimit.

As Whitehead's theorem is not true if the maps that appear in the diagram are not injective, it should not be expected that the functor $\underline{B}$ preserve colimits in full generality. 
Recall now that for any discrete group $G$ the fundamental group of $\underline{B} G$ can be identified (24], prop. 3) as the quotient of $G$ by the (normal) subgroup generated by the torsion elements. Using the main theorem 3.2 we can identify in some cases the universal cover of $\underline{\mathrm{B}} G$.

Proposition 4.4 Let $G$ be a discrete group which has a finite-dimensional model for $\underline{\mathrm{B}} G$, and let $T<G$ be the subgroup generated by the torsion elements. If the quotient $G / T$ is torsion-free, then the universal cover of $\underline{\mathrm{B}} G$ has the homotopy type of the $W_{\infty}$-nullification of $\mathrm{BT}$.

Proof We know that $T$ is normal in $G$, so we have a fibration

$$
\mathrm{B} T \longrightarrow \mathrm{B} G \longrightarrow \mathrm{B}(G / T) \text {. }
$$

As $G / T$ is torsion-free, its classifying space is $W_{\infty}$-null. Thus the previous fibration is preserved by $W_{\infty}$-nullification, and we obtain another one:

$$
\mathbf{P}_{W_{\infty}} \mathrm{B} T \longrightarrow \underline{\mathrm{B}} G \longrightarrow \mathrm{B}(G / T) .
$$

Note that, as $T$ is a subgroup of $G$, every model for $\underline{\mathrm{E}} G$ is also a model for $\underline{\mathrm{E}} T$. Hence, $\underline{\mathrm{B}} T$ is a model for $\mathbf{P}_{W_{\infty}} \mathrm{B} T$, and in particular $\pi_{1}\left(\mathbf{P}_{W_{\infty}} \mathrm{B} T\right)=\pi_{1}(\underline{\mathrm{B}} T)=$ $\{1\}$. This implies that $\mathbf{P}_{W_{\infty}} \mathrm{BT}$ is simply-connected, and we are done.

The last important consequence of the main theorem that we are going to prove here has to do with fibrations, and will have great importance in the remaining of this note.

It is a well-known fact of basic algebraic topology that if we have a group extension, then the sequence induced at the level of classifying spaces is a fibration sequence. Using the description of theorem [3.2] we find sufficient conditions that guarantee that the analogous result for $\underline{\mathrm{B}} G$ holds, and we show by means of an easy example that the statement does not need to be true if those hypotheses fail to be fulfilled.

So, suppose we have a short exact sequence of groups

$$
\{1\} \longrightarrow G_{1} \longrightarrow G \longrightarrow G_{2} \longrightarrow\{1\}
$$

which have a finite-dimensional model for the classifying space for proper bundles. Then the following result is true:

Proposition 4.5 If $G_{2}$ is torsion-free or $G_{1}$ admits a contractible model for $\underline{\mathrm{B}} G_{1}$, the homotopy fiber of the induced map $\underline{\mathrm{B}} G \longrightarrow \underline{\mathrm{B}} G_{2}$ is homotopy equivalent to $\underline{\mathrm{B}} G_{1}$. 
Proof It is enough to combine the results (13, 1.H.1 and 3.D.3) of DrorFarjoun with our description of $\underline{B} G$ as a nullification (Theorem 3.2 ).

The next example, that was one of the first motivations for our work, will show that the above conditions are necessary.

Example 4.6 Consider the group $D_{\infty} \times D_{\infty}$, and $H$ the index two subgroup whose elements are the words that can be written with an even number of letters. We have an extension

$$
H \longrightarrow D_{\infty} \times D_{\infty} \longrightarrow \mathbb{Z} / 2
$$

that induces a sequence of maps

$$
\underline{\mathrm{B}} H \longrightarrow \underline{\mathrm{B}}\left(D_{\infty} \times D_{\infty}\right) \longrightarrow \underline{\mathrm{B}} \mathbb{Z} / 2 .
$$

It is not hard to see that a model for $\underline{\mathrm{E}}\left(D_{\infty} \times D_{\infty}\right)$ is given by $\mathbb{R}^{2}$, and the quotient by the action of $\left(D_{\infty} \times D_{\infty}\right)$ is a square, which is contractible. By (24], prop. 8, see also lemma 6.1 below), $\underline{\mathrm{B} H}$ is homotopy equivalent to the 2 -sphere, and on the other hand, $\mathbb{Z} / 2$ is finite and so $\underline{B} \mathbb{Z} / 2$ is contractible. This means that the aforementioned sequence cannot be a fibration sequence. Of course, neither of the conditions of the proposition hold in this case.

Observe that $D_{\infty} \times D_{\infty}$ is a group of isometries of the plane. We will carefully study these groups in section 6 .

\section{Homotopy models of $\underline{\mathrm{B}} G$ for some classes of dis- crete groups}

In this section we will use the theorem 3.2 for describing the homotopy type of $\underline{\mathrm{B}} G$ for a wide range of groups. As a by-product, we will obtain that for every $G$ nilpotent such that it admits a finite-dimensional model for $\underline{\mathrm{B}} G, \underline{\mathrm{B}} G$ is nilpotent as a space, and we also determine, for $p$ odd, the $\mathrm{B} \mathbb{Z} / p$-nullification of classifying spaces of supersoluble groups. Let us start by considering the class of locally finite groups.

\subsection{Locally finite groups}

It is known that the classifying space for proper $G$-bundles of a group $G$ is contractible if the group $G$ is locally finite. We begin this section by presenting an easy proof of this fact in an ample range of cases. 
Proposition 5.1 Let $G$ be a locally finite group that admits a finite-dimensional model for $\underline{\mathrm{B}} G$. Then $\underline{\mathrm{B}} G$ is contractible.

Proof It is known (see for example 31, 9.8) that every locally finite group is the colimit of the directed system of its finite subgroups. Thus, we have a homotopy equivalence $\mathrm{B} G \simeq \operatorname{hocolim}_{\mathcal{C}} \mathrm{B} H$, where $\mathcal{C}$ is a contractible poset category (because it has an initial object given by the trivial group) and $\mathrm{B} H$ represents all the classifying spaces of finite groups $H$ of $G$. So, by ([13, 1.D.3), we obtain

$$
\mathbf{P}_{W_{\infty}} \mathrm{B} G \simeq \mathbf{P}_{W_{\infty}}\left(\text { hocolim }_{\mathcal{C}} \mathbf{P}_{W_{\infty}} \mathrm{B} H\right)=\mathbf{P}_{W_{\infty}}(|\mathcal{C}|)=\mathbf{P}_{W_{\infty}}(*)=*
$$

and we are done.

Remark 5.2 By [1], this result applies to all locally finite groups whose cardinal is smaller than $\aleph_{\omega}$.

Now we can prove the following result, that concerns to the classifying space for proper $G$-bundles of extensions of locally finite groups.

Proposition 5.3 Let

$$
\{1\} \longrightarrow K \longrightarrow G \longrightarrow Q \longrightarrow\{1\}
$$

be an extension of groups, $K$ a locally finite group whose cardinal is smaller than $\aleph_{\omega}$, and assume there is a bound on the order of finite subgroups of $Q$. Then if $Q$ admits a finite model for $\underline{\mathrm{B}} Q, G$ admits a finite model for $\underline{\mathrm{B}} G$, and then $\underline{\mathrm{B}} Q$ is homotopy equivalent to $\underline{\mathrm{B}} G$.

Proof If we apply the results 3.2 and 5.1 we obtain the statement is true if there is a finite-dimensional model for $\underline{\mathrm{B}} G$, and this happens by proposition 4.4 of 32 .

\subsection{Groups with the normalizer condition}

We study now the groups for which the normalizer condition holds. It is greatly remarkable that this class contains all the nilpotent groups.

Recall that a group $G$ is said to satisfy the normalizer condition if every proper subgroup of $G$ is distinct from its normalizer. In this case the following holds (see [22], page 215): 
(1) For every prime $p$, there exists a normal $p$-group $T_{p}$ such that if $x \in G$ and the order of $x$ is a power of $p$, then $x \in T_{p}$.

(2) The elements of finite order of $G$ form a normal subgroup of $G$ which is isomorphic to $\prod_{p \text { prime }} T_{p}$.

Throughout this section we will impose to the groups for which the normalizer condition holds that the torsion $p$-subgroups $T_{p}$ that we have just defined are locally finite. We need this condition because it is not known if the $\mathrm{B} \mathbb{Z} / p$ nullification of the classifying space of a general $p$-group is contractible if the group is not locally finite. Among the few examples that have been described of non-locally finite $p$-groups we can remark the Burnside groups $B(n, e)$ for $n>1$ and $e>664$ or the "monsters" of Tarski-Olshanskii. See [1] and 34] for more information about these families of groups.

All these facts have the following interesting consequence:

Proposition 5.4 If $G$ is a discrete group for which the normalizer condition holds, and $p_{1} \ldots p_{n}$ is a collection of primes, we have a homotopy equivalence $\mathbf{P}_{B \mathbb{Z} / p_{1} \vee \ldots \vee B \mathbb{Z} / p_{n}} \mathrm{~B} G \simeq \mathrm{B}\left(G / T_{p_{1}} \times \ldots \times T_{p_{n}}\right)$. In particular, there is an equivalence $\mathbf{P}_{W_{\infty}} \mathrm{B} G \simeq \mathrm{B}\left(G / \prod_{p \text { prime }} T_{p}\right)$.

Proof For simplicity, we will only prove the case of one prime $p$ (the generalization to a family is immediate). It is clear that $\mathrm{B} T_{p}$ is $\mathrm{B} \mathbb{Z} / p$-acyclic, and $\mathrm{B}\left(G / T_{p}\right)$ is $\mathrm{B} \mathbb{Z} / p$-null, so if we $\mathrm{B} \mathbb{Z} / p$-nullify the fibration

$$
\mathrm{B} T_{p} \longrightarrow \mathrm{B} G \longrightarrow \mathrm{B}\left(G / T_{p}\right)
$$

we obtain the desired homotopy equivalence.

If we suppose that $G$ is such that exists a finite-dimensional model for $\underline{B} G$ we have:

Corollary $5.5 \underline{\mathrm{B}} G \simeq \mathrm{B}\left(G / \prod_{p \text { prime }} T_{p}\right)$.

So we have a complete description of the homotopy type of $\underline{\mathrm{B}} G$.

Other case that can be solved with the same tools is the following:

Proposition 5.6 Let $G$ be a discrete group, $H$ a normal subgroup of $G$ for which the normalizer conditions holds, and such that $G / H$ does not have $p$ torsion. If $T_{p}$ is the $p$-torsion subgroup of $H$, then the $\mathrm{BZ} / p$-nullification of $\mathrm{B} G$ fits into the following fibration sequence:

$$
\mathrm{B}\left(H / T_{p}\right) \longrightarrow \mathbf{P}_{B \mathbb{Z} / p} \mathrm{~B} G \longrightarrow \mathrm{B}(G / H)
$$

and hence it is an Eilenberg-MacLane space. 
Proof The base of the fibration

$$
\mathrm{B} H \longrightarrow \mathrm{B} G \longrightarrow \mathrm{B}(G / H)
$$

is $\mathrm{BZ} / p$-null, so by $([13,3 . \mathrm{D} .3)$ the fibre is preserved under $\mathrm{B} \mathbb{Z} / p$-nullification. The result now follows from proposition 5.4

Taking into account the main theorem 3.2 the following corollary is immediate:

Corollary 5.7 In the hypotheses of the previous proposition, if $G / H$ is torsion-free, $T$ is the torsion subgroup of $H$ and there exists a finite-dimensional model for $\underline{B} G$, then the fibration

$$
\mathrm{B}(H / T) \longrightarrow \underline{\mathrm{B}} G \longrightarrow \mathrm{B}(G / H)
$$

defines the classifying space for proper $G$-bundles, which is again an EilenbergMacLane space.

We conclude this paragraph by focusing on nilpotent groups, that is a distinguished class of discrete groups for which the normalizer condition holds. The following result proves that the $\mathrm{B} \mathbb{Z} / p$-nullification preserves nilpotency when it is applied on classifying spaces of nilpotent groups, and in fact, the functor $\underline{B}$ sends nilpotent groups (for which the finiteness conditions hold) to nilpotent spaces.

Corollary 5.8 If $G$ is a nilpotent group, the nullification $\mathbf{P}_{B \mathbb{Z} / p_{1} \vee \ldots \vee B \mathbb{Z} / p_{n}} \mathrm{~B} G$ is, for every set of primes $\left\{p_{1}, \ldots, p_{n}\right\}$, the classifying space of a nilpotent group. If moreover $G$ admits a finite-dimensional model for $\underline{\mathrm{B}} G$, we obtain that $\underline{\mathrm{B}} G$ is again the classifying space of a nilpotent group, and hence nilpotent as a space.

Proof Using the previous results, it is enough recalling that the quotient of a nilpotent group is always nilpotent, and that according to (34], 2.7.1), every nilpotent $p$-group is locally finite.

In particular, using $(32,4.5)$ we have that the part of the previous corollary that alludes to $\underline{\mathrm{B}} G$ is always true if $G$ is a nilpotent group whose cardinal is smaller than $\aleph_{\omega}$ and whose torsion-free rank is finite. 


\subsection{Supersoluble groups}

In this paragraph we will compute, for $p$ odd, the $\mathrm{B} \mathbb{Z} / p$-nullification of classifying spaces of supersoluble groups. In this case we obtain no result about the homotopy type of $\underline{\mathrm{B}} G$ (for reasons that will be explained at the end) but we include here this computation because of its intrinsical interest, and because the way it has been worked out generalizes in some sense the methods we have used to compute the $\mathrm{B} \mathbb{Z} / p$-nullification in the previous sections.

Recall that a group $G$ is called supersoluble if it has cyclic normal series of finite length. It is known that every finitely generated nilpotent group is supersoluble, and that every supersoluble group is polycyclic.

Our key result for computing $\mathbf{P}_{B \mathbb{Z} / p} \mathrm{~B} G$ is the following ([35], page 67):

Proposition 5.9 If $G$ is a supersoluble group, there exists a characteristic series $1 \unlhd L \unlhd M \unlhd G$, in such a way that $L$ is finite with odd order, $M / L$ is a finitely-generated torsion-free nilpotent group and $G / M$ is a finite 2-group.

In the sequel we will use the notation of this proposition. Let $p$ be an odd prime, and consider the fibration:

$$
\mathrm{B} L \longrightarrow \mathrm{B} M \longrightarrow \mathrm{B}(M / L) \text {. }
$$

As $M / L$ is torsion-free, its classifying space is automatically $\mathrm{B} \mathbb{Z} / p$-null, and then by ([13, 3.D.3) we have the nullified fibration:

$$
\mathbf{P}_{B \mathbb{Z} / p} \mathrm{~B} L \longrightarrow \mathbf{P}_{B \mathbb{Z} / p} \mathrm{~B} M \longrightarrow \mathrm{B}(M / L) .
$$

Using ([17], 3.3), the fundamental group of $\mathbf{P}_{B \mathbb{Z} / p} \mathrm{~B} M$ is identified by an extension

$$
L / T_{\mathbb{Z} / p} L \longrightarrow \pi_{1} \mathbf{P}_{B \mathbb{Z} / p} \mathrm{~B} M \longrightarrow M / L
$$

where $T_{\mathbb{Z} / p} L$ is the minimal normal subgroup of $L$ that contains all the $p$ torsion (the $\mathbb{Z} / p$-radical), and the universal cover of $\mathbf{P}_{B \mathbb{Z} / p} \mathrm{~B} M$ is homotopy equivalent to $\mathbb{Z}[1 / p]_{\infty}\left(\mathrm{B} T_{\mathbb{Z} / p} L\right)$, where $\mathbb{Z}[1 / p]_{\infty}$ denotes Bousfield-Kan $\mathbb{Z}[1 / p]$ completion (see [6] for a definition).

Now, we have the fibration that involves $M$ and $G$ :

$$
\mathrm{B} M \longrightarrow \mathrm{B} G \longrightarrow \mathrm{B}(G / M) \text {. }
$$

This fibration is again preserved under $\mathrm{B} \mathbb{Z} / p$-nullification, because $G / M$ is a 2-group and $p$ is odd. The long exact sequence of the nullified fibration proves that the fundamental group of $\mathbf{P}_{B \mathbb{Z} / p} \mathrm{~B} G$ fits into the following exact sequence:

$$
\pi_{1} \mathbf{P}_{B \mathbb{Z} / p} \mathrm{~B} M \longrightarrow \pi_{1} \mathbf{P}_{B \mathbb{Z} / p} \mathrm{~B} G \longrightarrow G / M
$$


where the kernel has already been described. On the other hand, the universal cover of $\mathbf{P}_{B \mathbb{Z} / p} \mathrm{~B} G$ is the same as the universal cover of $\mathbf{P}_{B \mathbb{Z} / p} \mathrm{~B} M$ which is $\mathbb{Z}[1 / p]_{\infty} \mathrm{B} T_{\mathbb{Z} / p} L$, as we said before. Thus, we have described the desired nullification by means of a covering fibration. On the other hand, the fact that the classifying space of the quotient $G / M$ is not $\mathrm{B} \mathbb{Z} / 2$-null makes these methods useless for computing the $\mathrm{B} \mathbb{Z} / 2$-nullification of $\mathrm{B} G$. As an easy consequence of this, $\mathrm{B}(G / M)$ is not $W_{\infty}$-null (in the notation of theorem 3.2) and then we cannot get any homotopical description of $\underline{\mathrm{B}} G$ in this way.

\section{Nullifying classifying spaces of groups of isome- tries via proper actions}

So far we have applied theorem 3.2 for obtaining results about $\underline{B} G$ using properties of the nullification functors. In this section we will go the other way round, using geometric characteristics of the group $G$ for describing topological features of the classifying space.

Our analysis has been focused in some of the crystallographic groups of the plane, also known as wallpaper groups. Recall that these are groups of isometries of $\mathbb{R}^{2}$ that fix a pattern that is invariant under translations in the directions of two lineally independent vectors. It is known that they are exactly seventeen of these groups, and they are always finite extensions of $\mathbb{Z} \oplus \mathbb{Z}$ by a finite group. The main references available about the structure of these groups are [36] (that has been specially interesting for us because the big amount of pictures fundamental domains, mirror lines, rotation centers, generating regions, etc. that can be found on it), 26], 25], 10], [14] and [9]; we refer the reader to them for the details of the structure of the groups that in the sequel will stand without any explicit proof.

The general idea is to describe, for a prime $p$ and a discrete group $G$ that has $p$-torsion, the homotopy type of the $\mathrm{B} \mathbb{Z} / p$-nullification of the classifying space of $G$ using the main theorem 3.2. We have chosen wallpaper groups essentially for two reasons: the first of them is the following structure result, that is a particular case of proposition ([27, 5.2):

Lemma 6.1 Let $G$ be one of the seventeen wallpaper groups. Then $\mathbb{R}^{2}$, endowed with the natural action of $G$, is a model for $\underline{\mathrm{E}} G$.

The second feature of the wallpaper groups that we are going to use is that all of them possess a well-known model for the orbit space $\mathbb{R}^{2} / G$, which in fact 
is always described as a finite-dimensional orbifold. A list of these standard models can be found in [26]. According to the previous lemma, these spaces can be also interpreted as models for $\underline{\mathrm{B}} G$, and using this we will apply theorem 3.2 for obtaining the value of the $\mathrm{B} \mathbb{Z} / p$-nullification of the classifying space of $G$.

Now, if $G$ is a wallpaper group that only has torsion in a family of primes $P=\left\{p_{1} \ldots p_{r}\right\}$, it is an easy consequence of corollary 3.4 that every model for $\underline{\mathrm{B}} G$ is a model for $\mathrm{B}_{\mathcal{F}} G$, being $\mathcal{F}$ the family of finite subgroups of $G$ whose order is divided only for primes of the family $P$. In particular, if $G$ has torsion only in $P$ and admits a finite-dimensional model for $\underline{\mathrm{B}} G$, we have $\underline{\mathrm{B}} G=\mathrm{B}_{\mathcal{F}} G \simeq \mathbf{P}_{\mathrm{B} \mathbb{Z} / p_{1} \vee \ldots \vee \mathrm{B} \mathbb{Z} / p_{r}} \mathrm{~B} G$. We will frequently use this fact in the sequel.

We are going to study here three of the wallpaper groups, namely $\mathbf{p m m}, \mathbf{p} 3$ and $\mathbf{p 3 m 1} \mathbf{1}$. The main reason of our choice is that they give examples in which the $\mathrm{B} \mathbb{Z} / p$-nullification of the classifying space has homological dimension zero, positive and infinite, respectively.

(1) The discope group pmm As a group of symmetries of the real plane, this group is generated by two perpendicular translations and two reflections whose axes are also perpendicular, and in fact it is isomorphic to $D_{\infty} \times D_{\infty}$. Recall that this group already appeared in the construction of example 4.6 .

The group pmm contains reflections and rotations, and as it can be seen in the tables of [36], it tessellates the plane with rectangles. The orbit space of the plane by the action of this group is also a rectangle, and hence the classifying space for pmm-proper bundles is contractible. On the other hand, as the rotations that appear in the group are of order two, the group only contains torsion at the prime two, and thus $\underline{B} \mathbf{p m m}$ is a model for $\mathrm{B}_{\mathcal{F}_{2}} \mathrm{pmm}$. Now, according to the main theorem 3.2 we conclude that $\mathbf{P}_{B \mathbb{Z} / 2} \mathrm{Bpmm} \simeq *$.

(2) The tritrope group p3 This group is generated by two translations whose directions form an angle of $\pi / 3$ and a rotation of angle $2 \pi / 3$. A presentation with these generators is given by:

$$
\mathbf{p 3}=\left\{x, y, z ; x y x^{-1} y^{-1}=1, z^{3}=1, z x z^{-1} y^{-1} x=1, z y z^{-1} x=1\right\} .
$$

The distinguished isometries of this group are the 3-rotations, so we have no reflections nor glide-reflections and the torsion is concentrated in the prime three. The fundamental region of $\mathbf{p 3}$ (that is, the smallest region of $\mathbb{R}^{2}$ whose images under the action of $\mathbf{p} 3$ cover the plane) is a rhombus, and the action gives rise to a tessellation of $\mathbb{R}^{2}$ by hexagons; in fact, this is the simplest wallpaper 
group such that the induced tessellation is not by quadrilaterals. The quotient $\mathbb{R}^{2} / \mathbf{p} 3$ has then the shape of a non-slit turnover with three corners and no mirror points (see 25] for details), and in particular it has the homotopy type of a 2-sphere. Hence, using an analogous argument to that of the previous case, we obtain that the $\mathrm{B} \mathbb{Z} / 3$-nullification of $\mathrm{Bp3}$ is homotopy equivalent to $S^{2}$.

(3) The tryscope group p3m1 A convenient system of generators for this group is given by the two usual translations (whose directions form again an angle of $\pi / 3$ ), a rotation of angle $2 \pi / 3$, and a reflection whose axis is the bisectrix of the vectors that determine the generating translations; in particular, the reflection gives torsion in the prime two and the rotation gives it in the prime three.

On the other hand, maybe the best way to understand this group is as the group of reflections in the sides of an equilateral triangle. Hence, this is the fundamental region, and the lattice is hexagonal, as in the previous example. As one can see in [36], the orbit space by the action of $\mathbf{p} 3 \mathbf{m} \mathbf{1}$ on $\mathbb{R}^{2}$ is a triangle, and as this is a model for the classifying space for proper bundles, we have that Bp3m1 is contractible. Applying one more time theorem 3.2, we obtain that the $\mathrm{B} \mathbb{Z} / 2 \vee \mathrm{BZ} / 3$-nullification of the classifying space of $\mathbf{p 3 m 1}$ is a point.

Now we are also interested in the $\mathrm{B} \mathbb{Z} / 3$-nullification of Bp3m1, and we need to use a different strategy. The tryscope group can be seen as an extension of $\mathbb{Z} \oplus \mathbb{Z}$ by the symmetric group $\Sigma_{3}$, and a consequence of this is that p3 is an index two subgroup of $\mathbf{p} \mathbf{3 m 1} \mathbf{1}$. In particular, this gives rise to a fibration:

$$
\mathrm{Bp3} \longrightarrow \mathrm{Bp3m1} \longrightarrow \mathrm{B} \mathbb{Z} / 2 .
$$

The base space is $\mathrm{B} \mathbb{Z} / 3$-null, so according to (13, 3.D.3) and our previous description of $\mathbf{P}_{B \mathbb{Z} / 3} \mathrm{Bp3}$, the BZ/3-nullification of $\mathrm{Bp3m1}$ is identified by a covering fibration:

$$
S^{2} \longrightarrow \mathbf{P}_{\mathrm{B} \mathbb{Z} / 3} \mathrm{Bp3m1} \longrightarrow \mathrm{B} \mathbb{Z} / 2 .
$$

Now observe that the map $\mathrm{B} \mathbb{Z} / 3 \longrightarrow *$ is a $\mathbb{F}_{2}$-homology equivalence, and hence $H^{n}\left(\mathrm{Bp} 3 \mathbf{m} \mathbf{1} ; \mathbb{F}_{2}\right)$ is isomorphic to $H^{n}\left(\mathbf{P}_{\mathrm{B} \mathbb{Z} / 3} \mathrm{Bp3m1} ; \mathbb{F}_{2}\right)$. But as $\mathbf{p} 3 \mathbf{m} \mathbf{1}$ has 2-torsion, it has nontrivial $\mathbb{F}_{2}$-cohomology in arbitrarily high degrees, and then its BZ/3-nullification does, too. So, using universal coefficients theorem, we obtain that $\mathbf{P}_{\mathrm{BZ} / 3} \mathrm{Bp3m1}$ has infinite cohomological dimension. On the other hand, as $\underline{B} \mathbf{p} 3 \mathbf{m} 1$ is contractible and the rational homology of $\underline{\mathrm{B}} G$ is that of $\mathrm{B} G$ for any group $G$, the fact that $\mathrm{B} \mathbb{Z} / 3 \longrightarrow *$ is a $\mathbb{Q}$-homology equivalence implies that the rational homology of $\mathbf{P}_{\mathrm{B} \mathbb{Z} / 3} \mathrm{Bp3m1}$ is trivial. In particular, $\mathbf{P}_{\mathrm{B} \mathbb{Z} / 3} \mathrm{Bp3m1}$ is not homotopy equivalent to the product $S^{2} \times \mathrm{B} \mathbb{Z} / 2$. 
To conclude, note that in this context $S^{2}$ should be seen as two copies of the equilateral triangle glued along their edges, with the action of $\mathbb{Z} / 2$ swapping them. Hence, the previous fibration is not orientable (in the sense of [38], page 344). However, $\mathbf{P}_{\mathrm{B} \mathbb{Z} / 3} \mathrm{Bp3m1}$ cannot be homotopy equivalent to the projective plane, because this nullification has infinite cohomological dimension.

We think that the ideas developed in this section can give a lot of information about $\mathrm{B} \mathbb{Z} / p$-nullification of classifying spaces of groups of symmetries, and we plan to undertake in subsequent work its description for all the crystallographic (wallpaper and hyperbolic) groups, and also other groups of symmetries as rosette or frieze groups.

\section{The homotopy fiber of the natural map $\mathrm{B} G \longrightarrow$ $\mathrm{B}_{\mathcal{F}} G$}

We conclude this note by describing to what extent the homotopy fiber of the map $f: \mathrm{B} G \longrightarrow \mathrm{B}_{\mathcal{F}} G$ defined at the beginning of section 3 can be built using as pieces classifying spaces of subgroups of $G$ that belong to the family $\mathcal{F}$. To make this decomposition, the main tools that we are going to use are the left homotopy Kan extension of a functor and the Gabriel-Zisman localization. Now we will recall briefly these definitions.

In the sequel $\mathcal{C}$ and $\mathcal{D}$ will be small topological categories. Let $F: \mathcal{C} \rightarrow \mathcal{D}$ be a functor. If $d$ is an object of $\mathcal{D}$, then we define the overcategory $F \downarrow d$ as the category whose objects are pairs $(c, \phi)$ such that $c$ is an object of $\mathcal{C}$ and $\phi: F(c) \rightarrow d$ is a morphism in $\mathcal{D}$. A morphism between two pairs $(c, \phi)$ and $\left(c^{\prime}, \phi^{\prime}\right)$ is given by a map $\psi: c \rightarrow c^{\prime}$ in $\mathcal{C}$ such that $\phi(F(c))=\phi^{\prime} \circ F(\psi)\left(c^{\prime}\right)$. In the same way, the undercategory $d \downarrow F$ is defined as the category whose objects are pairs $(c, \phi)$ with $c \in \mathcal{C}$ and $\phi: c \rightarrow F(d)$ a morphism in $\mathcal{D}$. A morphism between $\psi:(c, \phi) \rightarrow\left(c^{\prime}, \phi^{\prime}\right)$ is a morphism $\psi^{\prime}: c \rightarrow c^{\prime}$ such that $F\left(\psi^{\prime}\right) \circ \phi=\phi^{\prime}$. When $F \downarrow d$ (respectively $d \downarrow F$ ) is contractible for every object $d$ in $\mathcal{D}$ we say that $F$ is left cofinal (respectively right cofinal).

Remark 7.1 The overcategory and undercategory are particular cases of "comma categories". For a complete study of the comma categories in the general context of category theory see (30], II.6).

From now on, and unless explicit mention against it, we will work in the category

Spaces of simplicial spaces (although most of the spaces that will appear will have a simplicial complex structure) 
Let $F: \mathcal{C} \rightarrow \mathcal{D}$ be a functor. Segal defined another functor, induced by $F$,

$$
L_{F}: \operatorname{Fun}(\mathcal{C}, \operatorname{Spaces}) \longrightarrow \operatorname{Fun}(\mathcal{D}, \operatorname{Spaces}),
$$

whose value on every $X: \mathcal{C} \rightarrow$ Spaces is given by $L_{F}(X)(d)=\operatorname{hocolim}_{F \downarrow d} X \circ p$, where $p$ is the projection functor $p: F \downarrow d \rightarrow \mathcal{C}$. The functor $L_{F}(X)$ is called the homotopy left Kan extension of $X$ along $F$.

The importance of that construction comes mainly from the next result:

Theorem 7.2 (Homotopy pushdown theorem) If $F: \mathcal{C} \rightarrow \mathcal{D}$ and $X: \mathcal{C} \rightarrow$ Spaces are functors, then there is a homotopy equivalence

$$
\operatorname{hocolim}_{\mathcal{D}} L_{F}(X) \simeq \operatorname{hocolim}_{\mathcal{C}} X .
$$

Proof The proof is done making use of the description of the homotopy left Kan extension as the classifying space of a category. See $([20,5.5)$.

Now we will recall the classical definition of localization of a category.

Theorem 7.3 (Gabriel-Zisman) Let $\mathcal{C}$ be a category. There exists another category $\mathcal{L}(\mathcal{C})$ and a functor $\mathcal{C} \longrightarrow \mathcal{L}(\mathcal{C})$ such that the following conditions hold:

- $\mathcal{L}$ inverts the morphisms of $\mathcal{C}$.

- If $F: \mathcal{C} \longrightarrow \mathcal{D}$ is another functor making the morphisms of $\mathcal{C}$ invertible, there exists one and only one functor $F^{\prime}: \mathcal{L}(\mathcal{C}) \longrightarrow \mathcal{D}$ such that $F^{\prime} \circ \mathcal{L}=$ $F$.

$\mathcal{L}(\mathcal{C})$ is called the category of fractions of $\mathcal{C}$ or simply the localization of $\mathcal{C}$.

Proof See (18], chapter 1).

Recall that if $X$ is a simplicial complex, the simplex category $\mathbf{\Gamma X}$ is the category whose objects are the simplices of $X$, and whose maps are the face maps (there are no nontrivial degeneracies). We will assume in the rest of the section that we will work with the model of $\mathrm{B}_{\mathcal{F}} G$ constructed in proposition 2.6. In the problem we are interested, $\boldsymbol{\Gamma} \mathbf{B}_{\mathcal{F}} \mathbf{G}^{o p}$ will play the role of $\mathcal{C}$, and $\mathcal{D}$ will be the localization of $\boldsymbol{\Gamma} \mathbf{B}_{\mathcal{F}} \mathbf{G}^{o p}$. From now on, we will use the model of $\mathrm{B}_{\mathcal{F}} G$ given in Proposition 2.6 and it is not hard to see that in this case, the $\mathbf{\Gamma} \mathbf{B}_{\mathcal{F}} \mathbf{G}$ is exactly the category $\boldsymbol{\Gamma}$ defined there. 
We have developed now all the ingredients we need, and we can give the decomposition, that is based in the concept of "homotopy average", proposed by Dror-Farjoun (see 13], chapter 9). So, consider the map $\mathrm{B} G \rightarrow \mathrm{B}_{\mathcal{F}} G$; if $S$ is the functor defined in section 2, call $\bar{S}$ the composition of $\mathrm{N}(S)$ with the Borel construction $\mathrm{E} G \times{ }_{G}(-)$. According to proposition 2.6 and (16, 6.5), we have that hocolim $\Gamma^{o p} \bar{S} \simeq \mathrm{B} G$. Now, if $\mathcal{L}$ is the localization functor previously defined, we can consider the left homotopy Kan extension $L_{\mathcal{L}}(\bar{S})$. The homotopy pushdown theorem 7.2 implies that we have a homotopy equivalence

$$
\operatorname{hocolim}_{\Gamma^{o p}} \bar{S} \simeq \operatorname{hocolim}_{\mathcal{L}\left(\boldsymbol{\Gamma}^{o p}\right)} L_{\mathcal{L}}(\bar{S}) .
$$

So joining all these data we obtain a string of maps

$$
\operatorname{hocolim}_{\mathcal{L}\left(\boldsymbol{\Gamma}^{o p}\right)} L_{\mathcal{L}}(\bar{S}) \simeq \operatorname{hocolim}_{\Gamma^{\mathrm{op}}} \bar{S} \simeq \mathrm{B} G \rightarrow \mathrm{B}_{\mathcal{F}} G .
$$

that, up to homotopy equivalence, is the map $\mathrm{B} G \longrightarrow \mathrm{B}_{\mathcal{F}} G$ that we are analyzing. So, we need to describe the inverse image of a simplex $\sigma$ of $\mathrm{B}_{\mathcal{F}} G$ in $\operatorname{hocolim}_{\mathcal{L}\left(\boldsymbol{\Gamma}^{o p}\right)} L_{\mathcal{L}}(\bar{S})$.

Consider first a simplex $\sigma \in \boldsymbol{\Gamma}$; it can be identified with a chain $G / H_{1} \rightarrow \ldots \rightarrow$ $G / H_{n}$, and then $\mathrm{N}(S)(\sigma)=G / H_{1}$ (as a discrete $G$-set). In a similar way, if

$$
\left\{G / H_{i_{1}} \rightarrow \ldots \rightarrow G / H_{i_{k}}\right\} \longrightarrow\left\{G / H_{1} \rightarrow \ldots \rightarrow G / H_{n}\right\}
$$

represents a morphism in $\boldsymbol{\Gamma}$, its image by $\mathrm{N}(S)$ is represented by the corresponding map $G / H_{1} \rightarrow G / H_{i_{1}}$. Now, the explicit definition over a simplex of $\mathrm{N}(S)$ is immediate.

Thus, if we identify now hocolim $\Gamma^{o p} \bar{S}$ with the Grothendieck construction of the nerve of $\bar{S}$, every simplex of hocolim $\Gamma^{o p} \bar{S}$ is represented $([16,6.5)$ by a triple $\left(\tau, \sigma_{1} \leq \ldots \leq \sigma_{n}, a H_{1}\right)$ where $\tau$ is a simplex of $\mathrm{E} G, \sigma_{i}$ is a simplex of $\boldsymbol{\Gamma}, g \in G$ and $\sigma_{n}=G / H_{1} \rightarrow \ldots \rightarrow G / H_{n}$. Observe that $\sigma_{1} \leq \ldots \leq \sigma_{n}$ represents a simplex in $\mathrm{N}(\boldsymbol{\Gamma})$, which is a model for $\mathrm{B}_{\mathcal{F}} G$, as we know. Now the map $\mathrm{B} G \longrightarrow \mathrm{B}_{\mathcal{F}} G$ that we are studying can be seen as the natural map

$$
\operatorname{hocolim}_{\Gamma^{o p}} \bar{S} \longrightarrow \operatorname{hocolim}_{\Gamma^{o p} *}
$$

induced by the natural transformation $\bar{S} \rightarrow *$. Hence the image of the previous triple under this map is the simplex $\sigma$, and according to the definition of $\bar{S}$ we can identify the inverse image of $\sigma$ with the pair $\left(\sigma, \bar{S}\left(\sigma_{n}\right)\right)$. Observe that, because of the definition of $\bar{S}$, the latter is the same as $(\sigma, \bar{S}(\sigma))$, which is in fact a model for the classifying space of $H_{1}$.

It remains to identify the image of the pair $\left(\sigma, \bar{S}\left(\sigma_{n}\right)\right)$ under the map

$$
\operatorname{hocolim}_{\Gamma^{o p}} \bar{S} \simeq \operatorname{hocolim}_{\mathcal{L}\left(\Gamma^{o p}\right)} L_{\mathcal{L}}(\bar{S})
$$


induced by the localization. As before, is is not hard to show that that every simplex of hocolim $\operatorname{L}_{\mathcal{L}\left(\boldsymbol{\Gamma}^{o p}\right)} L_{\mathcal{L}}(\bar{S})$ can be written as a pair $\left(\sigma_{1} \rightarrow \ldots \rightarrow\right.$ $\left.\sigma_{n}, L_{\mathcal{L}}(\bar{S})\left(\sigma_{1}\right) \rightarrow \ldots \rightarrow L_{\mathcal{L}}(\bar{S})\left(\sigma_{n}\right)\right)$, where $\sigma_{i} \rightarrow \sigma_{i+1}$ is a morphism in $\boldsymbol{\Gamma}$ or the formal inverse of a morphism in $\boldsymbol{\Gamma}$. Now, from the definition of $L_{\mathcal{L}}$ it can be deduced that the image of $(\sigma, \bar{S}(\sigma))$ is $\left(\sigma, L_{\mathcal{L}} \bar{S}(\sigma)\right)$. Note that in the latter we are looking at $\sigma$ in the localized category.

So we have checked that the inverse image of $\sigma$ by the string of equivalences (11) is exactly $\left(\sigma, L_{\mathcal{L}} \bar{S}(\sigma)\right)$. Hence, we can establish the following

Theorem 7.4 If $f: \mathrm{B} G \rightarrow \mathrm{B}_{\mathcal{F}} G$ is the map previously defined, then

$$
\mathbf{F i b}|f| \simeq\left|L_{\mathcal{L}} \bar{S}(\sigma)\right|
$$

for any simplex $\sigma$ of $\mathrm{B}_{\mathcal{F}} G$. Here || denotes the realization functor, and $\mathbf{F i b}|f|$ stands for the homotopy fiber of $|f|$.

Proof As the category $\boldsymbol{\Gamma}$ is itself constructed as a nerve, $\mathrm{N}(\boldsymbol{\Gamma})$ has structure of simplicial complex. So, every point of $\mathrm{N}(\boldsymbol{\Gamma})$ belongs to the interior of one and only one simplex of $\mathrm{N}(\boldsymbol{\Gamma})$, and it is enough to verify that the fibers of the simplices of $\mathrm{N}(\boldsymbol{\Gamma})$ are homotopy equivalent. On the other hand, a simplex is always contractible, so we need only check that the homotopy type of $\left|L_{\mathcal{L}} \bar{S}(\sigma)\right|$ does not depend on the simplex $\sigma$ of $\mathrm{B}_{\mathcal{F}} G$. We know, by the construction of the Kan extension, that $L_{\mathcal{L}}(\bar{S})(\sigma)=\operatorname{hocolim}_{\mathcal{L} \downarrow \sigma}(\bar{S} \circ p)$, where $p$ is the projection functor $p: \mathcal{L} \downarrow \sigma \rightarrow \mathcal{C}$. So, if $\sigma$ and $\sigma^{\prime}$ are two distinct simplices of $\mathrm{B}_{\mathcal{F}} G$, it is enough to see that the overcategories $\mathcal{L} \downarrow \sigma$ and $\mathcal{L} \downarrow \sigma^{\prime}$ are equivalent. In order to check this, let $g: \sigma \rightarrow \sigma^{\prime}$ be a morphism in $\mathcal{L}\left(\boldsymbol{\Gamma}^{o p}\right)$, that always exist because $\mathrm{B}_{\mathcal{F}} G$ is connected. In these conditions, $g$ induces a natural transformation

$$
\mathbf{T}_{g}: \mathcal{L} \downarrow \sigma \longrightarrow \mathcal{L} \downarrow \sigma^{\prime}
$$

that sends every object $(\tau, \phi)$ of $\mathcal{L} \downarrow \sigma$ to $(\tau, g \circ \phi) \in \mathcal{L} \downarrow \sigma^{\prime}$ and the morphisms to the obvious ones. But the morphism $g$ is invertible (because it is a morphism in the localized category), and clearly the natural transformations $\mathbf{T}_{g}$ and $\mathbf{T}_{g^{-1}}$ are inverses one of each other. In other words, the two overcategories are equivalent, and the corresponding homotopy colimits have the same homotopy type. So we are done.

The following corollary is immediate:

Corollary 7.5 The homotopy fiber Fib $|f|$ has the homotopy type of $\mid$ hocolim $_{\mathcal{L} \downarrow \sigma}(\bar{S} \circ p) \mid$, and in particular it is a homotopy colimit of classifying spaces of groups of $\mathcal{F}$ over a contractible category. 
We conclude this section by proving that the nerves of the two overcategories that appear in the proof of the previous theorem are contractible. We think that this question can have independent interest, and we would like to point out that, although the result seems to be known (see [13, 9.E.3), we have found no proof in the literature, so we give this one.

Proposition 7.6 Let $X$ be a simplicial complex, and let $\mathcal{L}: \mathbf{\Gamma X} \longrightarrow \mathcal{L}(\boldsymbol{\Gamma X})$ be the Gabriel-Zisman localization functor, where $\mathbf{\Gamma X}$ is the simplex category of $X$. Then for every simplex $\sigma \in X$ the overcategory $\mathcal{L} \downarrow \sigma$ is contractible.

Proof The idea of the proof is to build, for every simplex $\sigma \in X$ a homotopy between the identity map $\operatorname{Id}_{|\mathbf{N}(\mathcal{L} \downarrow \sigma)|}$ and a constant map. In order to do this, we will prove firstly the existence of a sequence of endofunctors

$$
\left\{F_{n}\right\}: \mathcal{L} \downarrow \sigma \longrightarrow \mathcal{L} \downarrow \sigma
$$

for every $n \geq 0$ such that $F_{0}=\operatorname{Id}$ and for every $(\tau, \phi) \in \mathcal{L} \downarrow \sigma$ there exists a natural number $n_{(\tau, \phi)}$ in such a way that $F_{m}((\tau, \phi))=(\sigma, \mathrm{Id})$ for every $m \geq n_{(\tau, \phi)}$.

In the sequel the maps in $\boldsymbol{\Gamma X}$ and their images in $\mathcal{L}(\boldsymbol{\Gamma X})$ will be denoted indistinctly by $i_{\alpha}$, where $\alpha$ will be an appropriate subindex. The inverse of $i_{\alpha}$ in the localized category will be called $j_{\alpha}$.

It is plain from the definition of the localization functor that every element $(\tau, \phi)$ of $\mathcal{L} \downarrow \sigma$ admits a unique expression of the form $\left(\tau, j_{n} \circ i_{n-1} \circ \ldots \circ j_{2} \circ i_{1}\right)$, where we allow that $j_{n}$ or $i_{1}$ can be the identity (but no one of the other maps that appear), $j_{t-1} \neq i_{t}^{-1} \neq j_{t+1}$ for every $t$.

So, we begin with $F_{0}=\mathrm{Id}$. Let us define the functor

$$
F_{1}: \mathcal{L} \downarrow \sigma \longrightarrow \mathcal{L} \downarrow \sigma .
$$

If $\left(\tau, j_{n} \circ i_{n-1} \circ \ldots \circ j_{2} \circ i_{1}\right)$ is an element of the overcategory, then we say $F_{1}\left(\left(\tau, j_{n} \circ i_{n-1} \circ \ldots \circ j_{2} \circ i_{1}\right)\right)=\left(i_{1}(\tau), j_{n} \circ i_{n-1} \circ \ldots \circ j_{2}\right)$, and the map induced by a face map will be sent to the identity map between the images. It is easy to see that this functor is well-defined.

Now, $F_{2}: \mathcal{L} \downarrow \sigma \longrightarrow \mathcal{L} \downarrow \sigma$ will be defined as $F_{2}\left(\left(\tau, j_{n} \circ i_{n-1} \circ \ldots \circ j_{2} \circ i_{1}\right)\right)=$ $\left(j_{2}^{-1} \circ i_{1}(\tau), j_{n} \circ i_{n-1} \circ \ldots \circ i_{3}\right)$. Observe that this is well-defined because the localization functor is, in this case, bijective over the objects. Again, the image of every morphism by $F_{2}$ will be the identity. It is clear again that this is a functor. 
In an analogous way, we can define, for $m$ odd, $F_{m}\left(\left(\tau, j_{n} \circ i_{n-1} \circ \ldots \circ j_{2} \circ i_{1}\right)\right)=$ $\left(i_{m} \circ j_{m-1} \circ i_{1}(\tau), j_{n} \circ i_{n-1} \circ \ldots j_{m+1}\right)$, and for $m$ even, $F_{m}\left(\left(\tau, j_{n} \circ i_{n-1} \circ \ldots \circ\right.\right.$ $\left.\left.j_{2} \circ i_{1}\right)\right)=\left(j_{m} \circ i_{m-1} \circ i_{1}(\tau), j_{n} \circ i_{n-1} \circ \ldots i_{m+1}\right)$, and the image sends every morphism to the identity map. This is the sequence we were looking for.

Our next goal will be to relate all these functors by natural transformations, in order to obtain the desired homotopy.

Let $m \geq 0$ be again a natural number. First we will define the transformation $T_{2 m}: F_{2 m} \longrightarrow F_{2 m+1}$. If $\left(\tau, j_{n} \circ i_{n-1} \circ \ldots \circ j_{2} \circ i_{1}\right)$ is an object of the overcategory, we define the map $F_{2 m}\left(\left(\tau, j_{n} \circ i_{n-1} \circ \ldots \circ j_{2} \circ i_{1}\right)\right) \longrightarrow F_{2 m+1}\left(\left(\tau, j_{n} \circ i_{n-1} \circ \ldots \circ\right.\right.$ $\left.\left.j_{2} \circ i_{1}\right)\right)$ as the obvious map induced by

$$
i_{2 m}: j_{2 m-1} \circ \ldots \circ i_{1}(\tau) \longrightarrow i_{2 m} \circ j_{2 m-1} \circ \ldots \circ i_{1}(\tau) .
$$

On the other hand, we define, for every $m \geq 1$, the natural transformation $T_{2 m-1}: F_{2 m} \longrightarrow F_{2 m-1}$ in the following way: $F_{2 m}\left(\left(\tau, j_{n} \circ i_{n-1} \circ \ldots \circ j_{2} \circ i_{1}\right)\right) \longrightarrow$ $F_{2 m-1}\left(\left(\tau, j_{n} \circ i_{n-1} \circ \ldots \circ j_{2} \circ i_{1}\right)\right)$ is the map induced by

$$
i_{2 m-1}: j_{2 m-1} \circ \ldots \circ i_{1}(\tau) \longrightarrow i_{2 m-2} \circ j_{2 m-3} \circ \ldots \circ i_{1}(\tau) .
$$

Recall the fact that, by definition of the $j$ 's, $j^{-1}$ represents a morphism in $\boldsymbol{\Gamma} \mathbf{X}$.

By the previous arguments we have defined a string of natural transformations

$$
\mathrm{Id}=F_{0} \stackrel{T_{0}}{\longrightarrow} F_{1} \stackrel{T_{1}}{\longleftarrow} F_{2} \stackrel{T_{2}}{\longrightarrow} F_{3} \stackrel{T_{3}}{\longleftarrow} \ldots
$$

Before we continue, we shall do a couple of remarks.

- It is known ([16], I.5) the functors $F_{n}$ define simplicial maps from nerves

$$
\mathrm{N}\left(F_{n}\right): \mathrm{N}(\mathcal{L} \downarrow \sigma) \longrightarrow \mathrm{N}(\mathcal{L} \downarrow \sigma)
$$

which, the same way, define maps $\left|f_{n}\right|$ from the realization of the nerve to itself. The fact that $F_{n}$ is always related to $F_{n+1}$ by a natural transformations means that $f_{n}$ is simplicially homotopic to $f_{n+1}$, and, in addition, $\left|f_{n}\right|$ is homotopic to $\left|f_{n+1}\right|$. The crucial point here is the homotopies between the realization of the maps are first defined over the vertices of the nerve of $\mathcal{L} \downarrow \sigma \times I$ (with the usual simplicial structure of the product) and then extended by linearity to all the complex. We will use this fact later.

- Let $\left(\tau, j_{n} \circ \ldots \circ i_{1}\right)$ be an object of the overcategory. From the definitions of the functors $F_{i}$ we can deduce that $F_{n} \circ \ldots \circ F_{1}\left(\left(\tau, j_{n} \circ \ldots \circ i_{1}\right)\right)=(\sigma, \mathrm{Id})$. So, as the chain of maps $j_{n} \circ \ldots \circ i_{1}$ is always finite, we can say that for every $(\tau, \phi) \in(\mathcal{L} \downarrow \sigma)$ there exists a minimal natural number $n_{(\tau, \phi)}$ such that $F_{n_{\tau, \phi}} \circ \ldots \circ F_{1}((\tau, \phi))=(\sigma$, Id $)$. At the level of nerves, we are saying that for every vertex $v \in \mathrm{N}(\mathcal{L} \downarrow \sigma)$ there exists $n_{v}$ such that $f_{n_{v}} \circ \ldots \circ f_{1}(v)=\mathrm{N}(\sigma, \mathrm{Id})$. 
For $n$ even, let us call $H_{n}$ the simplicial homotopy induced by the transformation $T_{n}$. If $n$ is odd, we call $H_{n-1}^{\prime}$ the homotopy induced by $T_{n}$ between $f_{n}$ and $f_{n-1}$, and put $H_{n-1}(x, t)=H_{n-1}^{\prime}(x, 1-t)$, the homotopy that begins in $f_{n-1}$ and ends at $f_{n}$.

Now we are prepared to define the homotopy between the identity and the constant map from the realization to itself with value $|\mathrm{N}(\sigma, \mathrm{Id})|$ (in the rest we will call this element $*)$. So, consider a vertex $v \in \mathrm{N}(\mathcal{L} \downarrow \sigma)$. We define a map $H:|\mathrm{N}(\mathcal{L} \downarrow) \sigma| \times I \longrightarrow|\mathrm{N}(\mathcal{L} \downarrow) \sigma|$ by

$$
H(v, t)= \begin{cases}\left|H_{0}\right|\left(v, n_{v} t\right) & \text { if } t \in\left[0, \frac{1}{n_{v}}\right] \\ \left|H_{1}\right|\left(v, n_{v} t-1\right) & \text { if } t \in\left[\frac{1}{n_{v}}, \frac{2}{n_{v}}\right] \\ \vdots & \vdots \\ \left|H_{n-1}\right|\left(v, n_{v} t-(n-1)\right) & \text { if } t \in\left[\frac{n_{v}-1}{n_{v}}, 1\right]\end{cases}
$$

The map $H$ defined in this way lineally extends to all of $|\mathrm{N}(\mathcal{L} \downarrow \sigma)|$. Let us see that $H$ is the desired map.

(1) If $v$ is a vertex of $\mathrm{N}(\mathcal{L} \downarrow \sigma), H(v, 0)=H_{0}(v, 0)=v$. In the same way, $H(v, 1)=H_{n_{v}}(v, 1)=*$. As $|H|_{i}$ is defined by linear extension for every $i$ and the same happens with $H$, the previous equalities hold for every point of the complex.

(2) $\quad H$ is continuous with respect to $t$ because the homotopies $\left|H_{i}\right|$ are, and $\left|H_{j}(x, 1)\right|=f_{j+1}(x)=\left|H_{j+1}(x, 0)\right|$ for every $x \in|\mathrm{N}(\mathcal{L} \downarrow \sigma)|$.

(3) Finally, $H$ is continuous respect the first component because it is defined by linear extension of a map defined on the vertices of a simplicial complex.

These three statements prove that $H$ is the homotopy between the identity and the constant map we were looking for. So, $\mathcal{L} \downarrow \sigma$ is contractible.

\section{References}

[1] S I Adyan, Problema Bernsaida i tozhdestva v gruppakh, [The Burnside problem and identities in groups], Izdat. "Nauka", Moscow (1975) MathReview

[2] G Z Arone, W G Dwyer, Partition complexes, Tits buildings and symmetric products, Proc. London Math. Soc. (3) 82 (2001) 229-256 MathReview

[3] P Baum, A Connes, N Higson, Classifying space for proper actions and $K$ theory of group $C^{*}$-algebras, from: " $C^{*}$-algebras: 1943-1993 (San Antonio, TX, 1993)", Contemp. Math. 167, Amer. Math. Soc. Providence, RI (1994) 240-291 MathReview 
[4] A K Bousfield, Localization and periodicity in unstable homotopy theory, J. Amer. Math. Soc. 7 (1994) 831-873 MathReview

[5] A K Bousfield, Homotopical localizations of spaces, Amer. J. Math. 119 (1997) 1321-1354 MathReview

[6] A K Bousfield, D M Kan, Homotopy limits, completions and localizations, Lecture Notes in Mathematics 304, Springer-Verlag, Berlin (1972) MathReview

[7] K S Brown, Cohomology of groups, Graduate Texts in Mathematics 87, Springer-Verlag, New York (1982) MathReview

[8] W Chachólski, On the functors $C W_{A}$ and $P_{A}$, Duke Math. J. 84 (1996) $599-$ 631 MathReview

[9] J H Conway, The orbifold notation for surface groups, from: "Groups, combinatorics \& geometry (Durham, 1990)", London Math. Soc. Lecture Note Ser. 165, Cambridge Univ. Press, Cambridge (1992) 438-447 MathReview

[10] H S M Coxeter, W O J Moser, Generators and relations for discrete groups, Second edition. Ergebnisse der Mathematik und ihrer Grenzgebiete, Neue Folge, Band 14, Springer-Verlag, Berlin (1965) MathReview

[11] W Dicks, P H Kropholler, IJ Leary, S Thomas, Classifying spaces for proper actions of locally finite groups, J. Group Theory 5 (2002) 453-480 MathReview

[12] T tom Dieck, Transformation groups, de Gruyter Studies in Mathematics 8, Walter de Gruyter \& Co. Berlin (1987) MathReview

[13] E Dror Farjoun, Cellular spaces, null spaces and homotopy localization, Lecture Notes in Mathematics 1622, Springer-Verlag, Berlin (1996) MathReview

[14] M P F du Sautoy, J J McDermott, G C Smith, Zeta functions of crystallographic groups and analytic continuation, Proc. London Math. Soc. (3) 79 (1999) 511-534 MathReview

[15] W G Dwyer, Homology decompositions for classifying spaces of finite groups, Topology 36 (1997) 783-804 MathReview

[16] W G Dwyer, H-W Henn, Homotopy theoretic methods in group cohomology, Advanced Courses in Mathematics. CRM Barcelona, Birkhäuser Verlag, Basel (2001) MathReview

[17] R J Flores, Nullification and cellularization of classifying spaces of finite groups, preprint, available at: http://hopf.math.purdue.edu/cgi-bin/generate?/Flores/draft1

[18] P Gabriel, M Zisman, Calculus of fractions and homotopy theory, Ergebnisse series 35, Springer-Verlag, New York (1967) MathReview

[19] P G Goerss, J F Jardine, Simplicial homotopy theory, Progress in Mathematics 174, Birkhäuser Verlag, Basel (1999) MathReview 
Nullification functors and the homotopy type of classifying spaces

[20] J Hollender, R M Vogt, Modules of topological spaces, applications to homotopy limits and $E_{\infty}$ structures, Arch. Math. (Basel) 59 (1992) 115-129 MathReview

[21] J F Jardine, Simplicial approximation, Theory Appl. Categ. 12 (2004) No. 2, 34-72 MathReview

[22] A.G. Kurosh, The theory of groups, vol. II, Chelsea publishing Co., 1960. LOOKUP

[23] J Lannes, L Schwartz, Sur la structure des A-modules instables injectifs, Topology 28 (1989) 153-169 MathReview

[24] I J Leary, B E A Nucinkis, Every CW-complex is a classifying space for proper bundles, Topology 40 (2001) 539-550 MathReview

[25] X Lee, http://www.xahlee.org/Wallpaper_dir/c5_17WallpaperGroups.html

[26] S Levy, http://www.geom.umn.edu/docs/reference/CRC-formulas/book.html

[27] W Lück, The type of the classifying space for a family of subgroups, J. Pure Appl. Algebra 149 (2000) 177-203 MathReview

[28] W Lück, Survey on classifying spaces for families of subgroups, Preprintreihe SFB 478 - Geometrische Strukturen in der Mathematik, Heft 308, Münster (2004)

[29] W Lück, R Stamm, Computations of $K$ - and L-theory of cocompact planar groups, K-Theory 21 (2000) 249-292 MathReview

[30] S MacLane, Categories for the working mathematician, Springer-Verlag, New York (1971) MathReview

[31] H Miller, The Sullivan conjecture on maps from classifying spaces, Ann. of Math. 120 (1984) 39-87 MathReview

[32] G Mislin, On the classifying space for proper actions, from: "Cohomological methods in homotopy theory (Bellaterra, 1998)", Progr. Math. 196, Birkhäuser, Basel (2001) 263-269 MathReview

[33] G Mislin, A Valette, Proper group actions and the Baum-Connes conjecture, Advanced Courses in Mathematics. CRM Barcelona. Birkhäuser Verlag, Basel (2003) MathReview

[34] A Y Ol'shanskiu,, Geometriya opredelyayushchikh sootnoshenii v gruppakh [The geometry of defining relations in groups], Sovremennaya Algebra [Modern Algebra], "Nauka", Moscow (1989) MathReview

[35] D Robinson, Finiteness conditions and generalized soluble groups, Ergebnisse series 62, Springer-Verlag, New York-Berlin (1972) MathReview

[36] D Schattschneider, The plane symmetry groups: their recognition and notation, Amer. Math. Monthly 85 (1978) 439-450 MathReview 
[37] J-P Serre, Cohomologie des groupes discrets, from: "Prospects in mathematics (Proc. Sympos. Princeton Univ. Princeton, NJ, 1970)", Princeton Univ. Press, Princeton, NJ (1971) 77-169. Ann. of Math. Studies, No. 70 MathReview

[38] R M Switzer, Algebraic topology - homotopy and homology, Grundlehren series 212, Springer-Verlag, New York (1975) MathReview

[39] R W Thomason, Homotopy colimits in the category of small categories, Math. Proc. Cambridge Philos. Soc. 85 (1979) 91-109 MathReview

Departamento de Matemáticas, Universidad Autónoma de Barcelona E-08193 Bellaterra, Spain

Email: ramonj@mat.uab.es

URL: http://mat.uab.es/ ramonj

Received: 25 November $2004 \quad$ Revised: 27 August 2005 